\title{
SYNTHESIS REACTIONS AND ANTIOXIDANT ACTIVITY OF SOME NEW HETEROCYCLES DERIVED FROM 2- ACETYLNAPHTHALENE
}

\author{
N.M. Taha, E. Islam, N.A. Marzouk, E. Mohamed and A. Ali \\ Department of Chemistry, Faculty of Science (Girls), Al-Azhar University, Nasr City, Cairo, \\ Egypt
}

\section{ABSTRACT :}

2-Methyl-4-(1-(naphthalen-2-yl)ethylidene)oxazol-5(4H)-one $\mathbf{2}$ was used as precursor for the preparation of some novel (1-(4-substituted)-2-methyl-4-(1-naphthalen-2yl)ethylidene)-1H-imidazol-5(4H)-one derivatives $\mathbf{4 a}, \mathbf{b}$ and other derivatives 3a,b, 5-12. Furthermore, the preparation of thieno[2,3-d]pyrimidin-4(3H)-one derivative 16,17 and 4iminothieno[2,3-d] pyrimidin-3-ylamine derivative 18, 19 is described starting from 2aminothiophen-3-carbonitrile derivative 15a. Some of the prepared products revealed a promising antioxidant activity by using 1,1-diphenyl-2,2-picryl hydrazyl free radical (DPPH) method.

Keywords: Imidazoles, thienopyrimidines, Antioxidant activity.

\section{INTRODUCTION :}

For a long time heterocyclic compounds have constituted one of the largest areas of research in organic chemistry. Heterocyclic compounds are of particular importance as they are associated with a wide variety of physiological activities attributed to heterocyclic systems known today. Among different nitrogen heterocycles, the imidazole ring which acts as a constituent of several important natural products, including purine, histamine, histidine and nucleic acid. Imidazoles and their derivatives have demonstrated a diverse set of biological activities such as antibacterial (Maddila, et al., 2010; Kumari, et al., 2010; Rajasekaran, et al., 2010; Nanda, et al., 2010; Ingle, et al., 2011; Shailesh, et al., 2012), anticancer (Li, et al., 2003, Mahboobi, et al., 2006; Chuu, et al., 2007; Hait, et al. 2009; Kanthou, et al., 2009; De Rychker, et al., 2009; Sanchila, et al., 2010; Wen-Tai, et al., 2010; Soroor, et al., 2012), anti-inflammatory (Achar, et al., 2010; Shailesh, et al., 2012), antitumor (Liberatore, et al., 2008), antioxidant (Maddila, et al., 2010; Rajasekaran, et al., 2010) activities. Also, prominent biological activities have been reported for fused thienopyrimidine derivatives (Hegab, et al., 2007; Shamrokh, et al., 2010; Marzouk, et al., 2011). In connection with our research program for the synthesis of different heterocyclic compounds, we describe here the synthesis of some new imidazole derivatives hopping to show promising antioxidant activity.

\section{RESULTS AND DISCUSSION}

The interaction of 2-acetylnaphthalene $\mathbf{1}$ with acetyl glycine in acetic anhydride and in the presence of fused sodium acetate effected cyclization and afforded the corresponding 2-methyl-4-(1-(naphthalen-2-yl)ethylidene)oxazol-5(4H)-one 2 (scheme 1). The structure of the oxazolinone derivative 2 was confirmed by elemental analysis and spectral data. The IR spectrum showed absorption band at $1668.9 \mathrm{~cm}^{-1}(\mathrm{C}=\mathrm{O})$; moreover, ${ }^{1} \mathrm{H}-\mathrm{NMR}$ spectrum exhibited signals at $\delta 2.05,2.70 \mathrm{ppm}$ for two methyl groups and $7.60-8.15$ for six aromatic protons, $8.47\left(\mathrm{~s}, 1 \mathrm{H}, \mathrm{C}_{-1}\right.$ naphthyl) and its mass spectrum afforded a molecular ion peak $\mathrm{M}^{+}$at $\mathrm{m} / \mathrm{z} 251(3.82 \%)$. The behaviour of oxazolinone derivative 2 towards some nucleophilic 
reagents was discussed under different conditions, as well as its transformation into the corresponding imidazolinone derivatives. Thus, interaction of $\mathbf{2}$ with p-nitroaniline or pchloroaniline in ethanol with boiling led to ring opening and gave the corresponding 2acetamido-N-substituted-3-(naphthalen-2-yl)but-2-enamides 3a,b which underwent heterocyclization by heating with acetic acid in the presence fused sodium acetate and produced imidazolinone derivatives $\mathbf{4 a , b}$ in $70 \%$ yield (scheme 1). On the other hand, compound 4a,b could be obtained in one step and better yield by direct reaction of $\mathbf{2}$ with pnitroaniline or $\mathrm{p}$-chloroaniline under reflux in acetic acid and fused sodium acetate $(89 \%$ yield) (scheme 1).

The structures of compounds $\mathbf{3}$ and $\mathbf{4}$ were deduced from elemental analysis and spectral data. IR spectra of $\mathbf{3}$ which showed absorption bands characteristic for $2 \mathrm{NH}$ groups while IR spectrum of $\mathbf{4}$ showed the absence of this absorption bands (c.f. exp.) Similarly, the reaction of oxazolinone $\mathbf{2}$ with secondary amines in ethanol or acetic acid led to ring opening to produce the corresponding acetamido compounds without transmitting into imidazolinone as in the case of primary amines. When compound 2 was treated with $\mathrm{N}, \mathrm{N}-$ diethylamine or morpholine in ethanol under reflux, the corresponding 2-acetamido-N,Ndiethyl-3-(naphthalen-2-yl)but-2-enamide $\mathbf{5}$ and N-1-morphoilino-3-naphthalen-2-yl)-1oxobut-2-en-2-yl)acetamide $\mathbf{6}$, were produced, respectively (scheme 1). The IR spectra of $\mathbf{5}$ and 6 showed characteristic absorption bands for $(2 \mathrm{C}=\mathrm{O})$ and $(\mathrm{NH})$ groups. Also ${ }^{1} \mathrm{HNMR}$ spectra $(\delta, \mathrm{ppm})$ revealed signals at $1.01\left(\mathrm{t}, \mathrm{J}=7.5 \mathrm{~Hz}, 6 \mathrm{H}, 2 \mathrm{CH}_{3}\right), 3.51\left(\mathrm{q}, \mathrm{J}=7.5 \mathrm{H}, 4 \mathrm{H}, 2 \mathrm{CH}_{2}\right)$ for compound 5 and for compound 6 revealed signals at $3.24-3.70\left(\mathrm{~m}, 8 \mathrm{H}, 4 \mathrm{CH}_{2}\right.$, morphilino) (c.f. exp.)

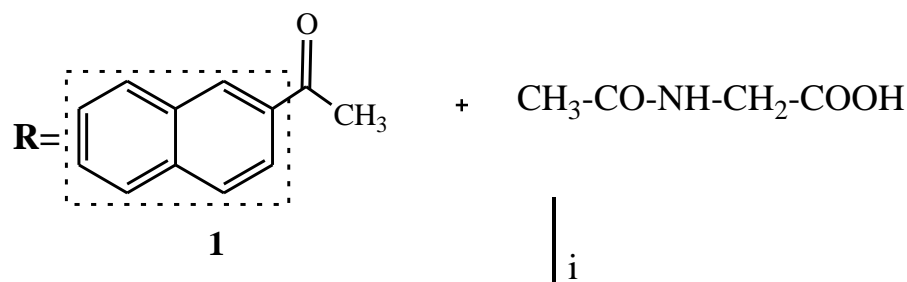<smiles>[R]C(C)=C(NC(=O)[CH]C(C)C)C(=O)NC(C(=O)N[Al])=C([R])C</smiles>

scheme 1 
Also, the behaviour of oxazolinone ring towards binucleophile reagent such hydrazine hydrate and phenylenediamine was studied hoping to prepare different heterocyclic of five and six membered ring hoping to have good pharmacological activity. Thus, interaction of oxazolinone $\mathbf{2}$ with hydrazine hydrate in ethanol under stirring at room temperature caused ring opening to give the corresponding hydrazide derivative 7 , while the product that isolated from the reaction of $\mathbf{2}$ with hydrazine hydrate in ethanol with boiling for $2 \mathrm{~h}$ was formulated as the triazine derivative $\mathbf{8}$ (scheme 2). The structures of compounds 7 and 8 were deduced from elemental analysis and spectral data. The ${ }^{1} \mathrm{H}-\mathrm{NMR}$ spectrum of compound $7(\delta, \mathrm{ppm})$ reaveled signals at $3.72\left(\mathrm{br}, 2 \mathrm{H}, \mathrm{NH}_{2}\right), 9.25,9.51(2 \mathrm{~s}, 2 \mathrm{H}, 2 \mathrm{NH})$ exchangeable with $\mathrm{D}_{2} \mathrm{O}$, while mass spectrum of compound $\mathbf{8}$ exhibited a molecular ion peak at $\mathrm{m} / \mathrm{z}=265(18.67 \%)$ (c.f. exp.).

Moreover, interaction of oxazolinone 2 with p-phenylenediamine in ethanol consumed one mole of oxazolinone and produce 2-acetamido- $\mathrm{N}$-(4-aminophenyl)-3(naphthalen-2-yl)but-2-enamide 9 which under-went heteocyclization by heating with acetic acid in the presence fused sodium acetate and produced imidazolinone derivative $\mathbf{1 0}$ (scheme 2). The proposed structures of compounds $\mathbf{9}$ and $\mathbf{1 0}$ was confirmed by elemental analysis and spectral data (c.f. exp.). Treatment of the later compound with p-toluene sulphonyl chloride or p-chlorobenzaldehyde afforded the corresponding sulfonamide derivative $\mathbf{1 1}$ and the Schiff base 12, respectively. Inspection of the IR spectrum of the reaction product $\mathbf{1 1}$ revealed absorption bands characteristic for $(\mathrm{NH}),(\mathrm{C}=\mathrm{O})$ groups at 3432,1671 and $\left(\mathrm{SO}_{2}\right)$ group at $1187,1361 \mathrm{~cm}^{-1} ;{ }^{1} \mathrm{H}-\mathrm{NMR}$ spectrum showed signals $(\delta, \mathrm{ppm})$ at $1.22,1.52,2.57(3 \mathrm{~s}$, $\left.9 \mathrm{H}, 3 \mathrm{CH}_{3}\right)$, and $7.25\left(\mathrm{~s}, 1 \mathrm{H}, \mathrm{NH}\right.$, exchangeable with $\left.\mathrm{D}_{2} \mathrm{O}\right)$, while for compound 12 the ${ }^{1} \mathrm{H}-$ NMR spectrum revealed a signal at $9.83 \mathrm{ppm}$ characteristic for $(-\mathrm{CH}=\mathrm{N}-)$ (c.f. exp.).

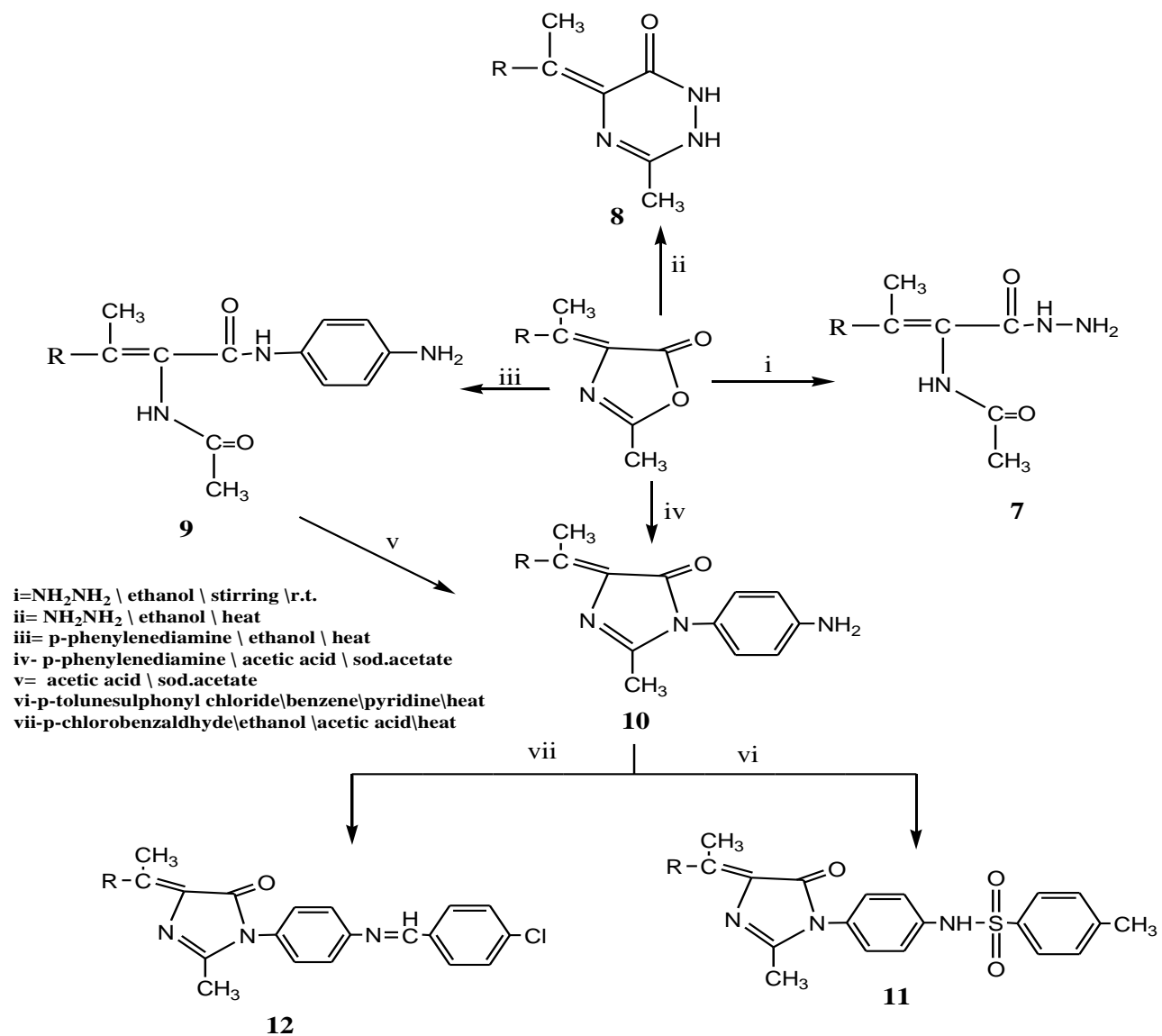

Scheme 2 
It was reported (Hessien, et al., 2009; Hafez, et al., 2010) that various activated nitriles and enaminonitriles were used as intermediates for the syntheses of thieno derivatives and thienopyrimidine derivatives. Thus, condensation of 2-acetyl naphthalene 1 with malononitrile in ethanolic-piperidine solution under reflux gave the corresponding ethylidinemalononitrile derivative 13. IR spectrum showed sharp absorption bands at 2210 and 1589 characteristic for $(2 \mathrm{C} \equiv \mathrm{N})$ group and $(\mathrm{C}=\mathrm{C})$, respectively and the MS spectrum showed a molecular ion peak at $\mathrm{m} / \mathrm{z}=218\left(\mathrm{M}^{+}, 100 \%\right)$. Treatment of $\mathbf{1 3}$ with elemental sulfur under Gewald reaction conditions (Gewald, 1965) furnished 2-aminothiophene-3carbonitrile derivatives 15a. The formation of compound 15a occurred via thiation of methyl group in compound $\mathbf{1 3}$ to give $\mathbf{1 4}$ as an intermediate followed by intramolecualr cyclization (scheme 3). Compound 15a was also obtained directly by interaction of ketone 1 with a mixture of malononitrile and elemental sulfur in the presence of few drops of triethylamine. The obtained product 15a through out the two pathways was checked by TLC and mixed m.p which showed no depression.

Similarly, when compound $\mathbf{1}$ was treated ethyl cyanoacetate and elemental sulfur under Gewald reaction conditions (Gewald, 1965) gave the corresponding ethyl-2aminothiophen-3-carboxylate derivative 15b (scheme 3). The IR spectra showed absorption bands $\left(\mathrm{v}, \mathrm{cm}^{-1}\right)$ at $2206(\mathrm{C} \equiv \mathrm{N})$ and $3202 \& 3322\left(\mathrm{NH}_{2}\right)$ groups for 15a while for $\mathbf{1 5 b}$ showed $(\mathrm{C}=\mathrm{O})$ at 1721 and $\mathrm{NH}_{2}$ at 3200, 3321 (c.f. exp.)

The 4-pyrmidinone derivative was prepared by reacting compound 15a with formic acid under reflux to give derivative 16 (scheme 3). Product 16 was formed presumably via intermediacy of the corresponding oxazinimine derivative (Abdelrazek, et al., 1996; Hegab, et al., 2007) which then rearranged under the conditions of the reaction. On the other hand, when compound 15a was refluxed with triethyl orthoformate, it afforded derivative 17. When the ethanolic solution of the later compound was stirred at room temperature with hydrazine hydrate, it afforded 4-iminopyrimidin-3-ylamine derivative 18. the structures of compounds 16-18 were deduced from elemental analysis and spectral data. IR spectra showed the absence of cyano group for $\mathbf{1 6}$ and 18, while ${ }^{1} \mathrm{H}-\mathrm{NMR}$ spectrum revealed signals characteristic for ethyl group for 17 (c.f. exp.). However, the pyrmidin-4-ylhydrazine derivative 19 was obtained by treatment of compound 17 with hydrazine hydrate under reflux (scheme 3). Also, compound $\mathbf{1 8}$ was isomerized to corresponding more stable 4hydrazino derivative $\mathbf{1 9}$ upon reflux in ethanol in the presence of hydrazine hydrate. Actually, hydrazine hydrate acts as a base in this Dimroth type of rearrangement, which involves a sequence of ring opening and ring closure reaction (Mohamed, et al., 2005; Rashad, et al., 2005; Hegab et al., 2007). 


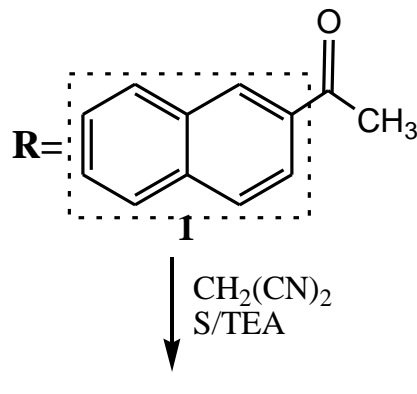<smiles>[R]c1csc(N)c1Br</smiles>

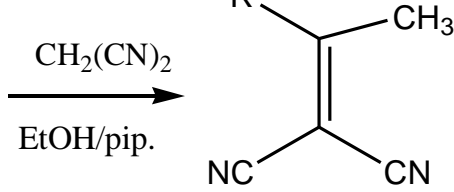

13

S/TEA

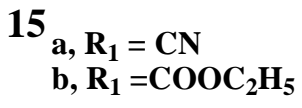<smiles>[R]c1csc(/N=C\OCC)c1C#N</smiles><smiles>[R]c1csc2ncn(N)c(=N[14CH3])c12</smiles>

18

Scheme 3

\section{Antioxidant activity.}

\section{RESULTS:}

The provided compounds showed different antioxidant activity using 1,1-diphenyl2,2-picryl hydrazyl free radical (DPPH) radical scavenging method compared to ascorbic acid standard as shown by the following table : 
Table I . Antioxidant activity of some synthesized compounds

\begin{tabular}{|c|c|c|}
\hline & Compound No. & DDPH radical scavenging Activity \\
\hline & $3 \mathbf{a}$ & + \\
\hline & $3 \mathbf{b}$ & + \\
\hline & 5 & +++ \\
\hline & 6 & + \\
\hline & 7 & + \\
\hline & 8 & ++ \\
\hline & 9 & + \\
\hline & 10 & + \\
\hline & 11 & + \\
\hline & 12 & +++ \\
\hline+ & Weak & \\
\hline++ & Moderate & \\
\hline+++ & Good & \\
\hline++++ & Strong & \\
\hline & No activity & \\
\hline
\end{tabular}

Comment : Qualitative analysis are recommended for the promising result. 
Table II: Physical data of the new synthesized compounds

\begin{tabular}{|c|c|c|c|c|c|c|c|}
\hline \multirow{2}{*}{$\begin{array}{l}\text { Compd. } \\
\text { No. }\end{array}$} & \multirow[t]{2}{*}{$\overline{\text { M.P. }\left[{ }^{\circ} \mathrm{C}\right]}$} & \multirow{2}{*}{$\begin{array}{c}\text { Yield[\%] } \\
\text { solvent }\end{array}$} & \multirow{2}{*}{$\begin{array}{c}\text { Mol. } \\
\text { Formula/Mol.wt. }\end{array}$} & \multicolumn{4}{|c|}{ (Calcd./Found)\% } \\
\hline & & & & $\mathrm{C}$ & $\mathrm{H}$ & $\mathrm{N}$ & $\mathrm{Cl}, \mathrm{S}$, \\
\hline 2 & $78-79$ & $\begin{array}{c}80 \\
\text { Pet.E 40-60 }\end{array}$ & $\begin{array}{c}\mathrm{C}_{16} \mathrm{H}_{13} \mathrm{NO}_{2} \\
251.28\end{array}$ & $\begin{array}{l}76.48 \\
76.51\end{array}$ & $\begin{array}{l}5.21 \\
5.19\end{array}$ & $\begin{array}{l}5.57 \\
5.54\end{array}$ & -- \\
\hline $\mathbf{3 a}$ & $105-107$ & $\begin{array}{l}85 \\
B\end{array}$ & $\begin{array}{c}\mathrm{C}_{22} \mathrm{H}_{19} \mathrm{~N}_{3} \mathrm{O}_{4} \\
389.4\end{array}$ & $\begin{array}{l}67.86 \\
67.83\end{array}$ & $\begin{array}{l}4.92 \\
4.91\end{array}$ & $\begin{array}{l}10.79 \\
10.77\end{array}$ & \\
\hline $\mathbf{3 b}$ & $200-202$ & $\begin{array}{l}80 \\
\mathrm{D}\end{array}$ & $\begin{array}{c}\mathrm{C}_{22} \mathrm{H}_{19} \mathrm{ClN}_{2} \mathrm{O}_{2} \\
378.85\end{array}$ & $\begin{array}{l}69.75 \\
69.73\end{array}$ & $\begin{array}{l}5.05 \\
5.07\end{array}$ & $\begin{array}{l}7.39 \\
7.41\end{array}$ & $\begin{array}{l}9.36 \\
9.31 \\
(\mathrm{Cl})\end{array}$ \\
\hline $4 a$ & $115-116$ & $\begin{array}{l}87 \\
\mathrm{E}\end{array}$ & $\begin{array}{c}\mathrm{C}_{22} \mathrm{H}_{17} \mathrm{~N}_{3} \mathrm{O}_{3} \\
371.39\end{array}$ & $\begin{array}{l}71.15 \\
71.17\end{array}$ & $\begin{array}{l}4.61 \\
4.65\end{array}$ & $\begin{array}{l}11.31 \\
11.37\end{array}$ & \\
\hline $4 b$ & $210-211$ & $\begin{array}{l}70 \\
B\end{array}$ & $\begin{array}{c}\mathrm{C}_{22} \mathrm{H}_{17} \mathrm{ClN}_{2} \mathrm{O} \\
360.84\end{array}$ & $\begin{array}{l}73.23 \\
73.25\end{array}$ & $\begin{array}{l}4.75 \\
4.72\end{array}$ & $\begin{array}{l}7.76 \\
7.79\end{array}$ & $\begin{array}{l}9.83 \\
(\mathrm{Cl})\end{array}$ \\
\hline 5 & $110-112$ & $\begin{array}{l}95 \\
\mathrm{~B}\end{array}$ & $\begin{array}{c}\mathrm{C}_{20} \mathrm{H}_{24} \mathrm{~N}_{2} \mathrm{O}_{2} \\
324.42\end{array}$ & $\begin{array}{l}74.04 \\
74.06\end{array}$ & $\begin{array}{l}7.46 \\
7.43\end{array}$ & $\begin{array}{l}8.64 \\
8.60\end{array}$ & -- \\
\hline 6 & $50-51$ & $\begin{array}{c}90 \\
\text { Pet.E }\end{array}$ & $\begin{array}{c}\mathrm{C}_{20} \mathrm{H}_{22} \mathrm{~N}_{2} \mathrm{O}_{3} \\
338.4\end{array}$ & $\begin{array}{l}70.99 \\
70.97\end{array}$ & $\begin{array}{l}6.55 \\
6.57\end{array}$ & $\begin{array}{l}8.28 \\
8.27\end{array}$ & -- \\
\hline 7 & 180-182 & $\begin{array}{l}70 \\
M\end{array}$ & $\begin{array}{c}\mathrm{C}_{16} \mathrm{H}_{17} \mathrm{~N}_{3} \mathrm{O}_{2} \\
283.33\end{array}$ & $\begin{array}{l}67.83 \\
67.86\end{array}$ & $\begin{array}{l}6.05 \\
6.02\end{array}$ & $\begin{array}{l}14.83 \\
14.85\end{array}$ & -- \\
\hline 8 & $155-156$ & $\begin{array}{l}85 \\
M\end{array}$ & $\begin{array}{c}\mathrm{C}_{16} \mathrm{H}_{15} \mathrm{~N}_{3} \mathrm{O} \\
265.31\end{array}$ & $\begin{array}{l}72.43 \\
72.41\end{array}$ & $\begin{array}{l}5.70 \\
5.69\end{array}$ & $\begin{array}{l}15.84 \\
15.81\end{array}$ & -- \\
\hline 9 & $120-121$ & $\begin{array}{l}75 \\
\mathrm{~B}\end{array}$ & $\begin{array}{c}\mathrm{C}_{22} \mathrm{H}_{21} \mathrm{~N}_{3} \mathrm{O}_{2} \\
359.42\end{array}$ & $\begin{array}{l}73.52 \\
73.49\end{array}$ & $\begin{array}{l}5.89 \\
5.91 \\
\end{array}$ & $\begin{array}{l}11.69 \\
11.71 \\
\end{array}$ & -- \\
\hline 10 & $210-212$ & $\begin{array}{l}75 \\
\mathrm{M}\end{array}$ & $\begin{array}{c}\mathrm{C}_{22} \mathrm{H}_{19} \mathrm{~N}_{3} \mathrm{O} \\
341.41\end{array}$ & $\begin{array}{l}77.40 \\
77.47\end{array}$ & $\begin{array}{l}5.61 \\
5.59\end{array}$ & $\begin{array}{l}12.31 \\
12.29\end{array}$ & -- \\
\hline 11 & $50-51$ & $\begin{array}{c}85 \\
\text { Pet.E } \\
40-60\end{array}$ & $\begin{array}{c}\mathrm{C}_{29} \mathrm{H}_{25} \mathrm{~N}_{3} \mathrm{O}_{3} \mathrm{~S} \\
495.59\end{array}$ & $\begin{array}{l}70.28 \\
70.25\end{array}$ & $\begin{array}{l}5.08 \\
5.11\end{array}$ & $\begin{array}{l}8.48 \\
8.46\end{array}$ & $\begin{array}{c}6.47 \\
6.51 \\
(\mathrm{~S})\end{array}$ \\
\hline 12 & $70-71$ & $\begin{array}{l}80 \\
\mathrm{~B}\end{array}$ & $\begin{array}{c}\mathrm{C}_{29} \mathrm{H}_{22} \mathrm{~N}_{3} \mathrm{ClO} \\
463.96\end{array}$ & $\begin{array}{l}75.07 \\
75.11\end{array}$ & $\begin{array}{l}4.78 \\
4.77\end{array}$ & $\begin{array}{l}9.06 \\
9.03\end{array}$ & $\begin{array}{l}7.64 \\
7.62 \\
(\mathrm{Cl})\end{array}$ \\
\hline 13 & $78-79$ & $\begin{array}{l}80 \\
B\end{array}$ & $\begin{array}{c}\mathrm{C}_{15} \mathrm{H}_{10} \mathrm{~N}_{2} \\
218.25\end{array}$ & $\begin{array}{l}82.55 \\
82.53\end{array}$ & $\begin{array}{l}4.62 \\
4.66\end{array}$ & $\begin{array}{l}12.84 \\
12.85\end{array}$ & -- \\
\hline $15 a$ & $120-121$ & $\begin{array}{l}85 \\
\mathrm{~B}\end{array}$ & $\begin{array}{c}\mathrm{C}_{15} \mathrm{H}_{10} \mathrm{~N}_{2} \mathrm{~S} \\
250.32\end{array}$ & $\begin{array}{l}71.97 \\
71.91\end{array}$ & $\begin{array}{l}4.03 \\
4.05\end{array}$ & $\begin{array}{l}11.19 \\
11.21\end{array}$ & $\begin{array}{c}12.81 \\
12.85 \\
\text { (S) }\end{array}$ \\
\hline$\overline{15 b}$ & $45-46$ & $\begin{array}{c}55 \\
\text { Pet-E } \\
40-60\end{array}$ & $\begin{array}{c}\mathrm{C}_{17} \mathrm{H}_{15} \mathrm{NO}_{2} \mathrm{~S} \\
297.37\end{array}$ & $\begin{array}{l}68.66 \\
68.63\end{array}$ & $\begin{array}{l}5.08 \\
5.09\end{array}$ & $\begin{array}{l}4.71 \\
4.69\end{array}$ & $\begin{array}{c}10.78 \\
10.81 \\
(\mathrm{~S})\end{array}$ \\
\hline 16 & $220-222$ & $\begin{array}{l}70 \\
E\end{array}$ & $\begin{array}{c}\mathrm{C}_{16} \mathrm{H}_{10} \mathrm{~N}_{2} \mathrm{OS} \\
278.33\end{array}$ & $\begin{array}{l}69.04 \\
69.05\end{array}$ & $\begin{array}{l}3.62 \\
3.60\end{array}$ & $\begin{array}{l}10.06 \\
10.08\end{array}$ & $\begin{array}{c}11.52 \\
11.58 \\
(\mathrm{~S})\end{array}$ \\
\hline 17 & $165-166$ & $\begin{array}{l}70 \\
B\end{array}$ & $\begin{array}{c}\mathrm{C}_{18} \mathrm{H}_{14} \mathrm{~N}_{2} \mathrm{OS} \\
306.38\end{array}$ & $\begin{array}{l}70.56 \\
70.60\end{array}$ & $\begin{array}{l}4.61 \\
4.69\end{array}$ & $\begin{array}{l}9.14 \\
9.10\end{array}$ & $\begin{array}{c}10.47 \\
10.42 \\
(\mathrm{~S})\end{array}$ \\
\hline 18 & 238 & $\begin{array}{l}75 \\
D\end{array}$ & $\begin{array}{c}\mathrm{C}_{16} \mathrm{H}_{12} \mathrm{~N}_{4} \mathrm{~S} \\
292.36\end{array}$ & $\begin{array}{l}65.73 \\
65.74\end{array}$ & $\begin{array}{l}4.14 \\
4.15\end{array}$ & $\begin{array}{l}19.16 \\
19.19\end{array}$ & $\begin{array}{l}10.97 \\
10.99\end{array}$ \\
\hline 19 & $>260$ & $\begin{array}{l}70 \\
\mathrm{D}\end{array}$ & $\begin{array}{c}\mathrm{C}_{16} \mathrm{H}_{12} \mathrm{~N}_{4} \mathrm{~S} \\
292.36\end{array}$ & $\begin{array}{l}65.73 \\
65.75\end{array}$ & $\begin{array}{l}4.14 \\
4.17\end{array}$ & $\begin{array}{l}19.16 \\
19.14\end{array}$ & $\begin{array}{l}10.97 \\
10.93\end{array}$ \\
\hline
\end{tabular}

Solvent of crystallization : Pet.E: petroleum ether; B : Benzene; D: dioxan ; M : Methanol ; E : Ethanol 


\section{Experimental}

Melting points were recorded on an electrothermal IA 9100 digital melting point apparatus and were uncorrected. IR spectra $\left(\mathrm{V}_{\max }\right.$ in $\left.\mathrm{cm}^{-1}\right)$ were recorded on a Shimadzu FTIR 8300 spectrophotometer using $\mathrm{KBr}$ pellets technique. ${ }^{1} \mathrm{H}-\mathrm{NMR}$ and ${ }^{13} \mathrm{C}-\mathrm{NMR}$ spectra were recorded using Bruker WM-400 spectrophotometer using DMSO- $\mathrm{d}_{6}$ as the solvent and TMS as the internal reference (chemist shifts in ppm). The mass spectra were run at $70 \mathrm{eV}$ with a finnigan SSQ7000 spectrophotometer (thermo-instrument system incorporation, USA) Elemental analysis were operated using Mario El Mentar apparatus, Organic microanalysis unit. Elemental analysis and the above spectra were measured the at National Research Center. Pharmacology was carried out in the Regional Center for Mycology \& Biotechnology, Al-Azhar University.

\section{2-Methyl-4-(1-naphthalen-2-yl)ethylidene)oxazol-5-(4H)-one (2).}

A mixture of compound $1(0.01 \mathrm{~mol})$, acetyl glycine $(0.01 \mathrm{~mol})$ and $2 \mathrm{gm}$ of fused sodium acetate in $(10 \mathrm{~mL})$ of acetic acid and $5 \mathrm{~mL}$ of acetic anhydride was refluxed for $1 \mathrm{~h}$. The reaction mixture was allowed to cool and then poured into cold water, the product obtained was filtered, dried and recrystallized to give 2. IR $\left(\mathrm{cm}^{-1}, \mathrm{v}\right): 1668.9(\mathrm{C}=\mathrm{O}) ;{ }^{1} \mathrm{H}-$ NMR (DMSO-d 6 , $\delta$, ppm) 2.05, $2.70\left(2 \mathrm{~s}, 6 \mathrm{H}, 2 \mathrm{CH}_{3}\right), 7.60-8.15(\mathrm{~m}, 6 \mathrm{H}, \mathrm{ArH}), 8.47(\mathrm{~s}, 1 \mathrm{H}, \mathrm{C}$ $\left.{ }_{1} \mathrm{ArH}\right) ; \quad \mathrm{MS}: \mathrm{m} / \mathrm{z}(\%): 251\left[\mathrm{M}^{+}, 3.82\right]$.

General procedure for the preparation of the compounds 3a,b,5,6,8 and 9 .

To a solution of oxazolinon-5-one $(0.01 \mathrm{~mol})$ in ethanol $(30 \mathrm{~mL})$, the request amine (p-nitroaniline, p-chloroaniline, diethylamine, morpholine, hydrazine hydrate or $\mathrm{p}$ aminoaniline $(0.01 \mathrm{~mol})$ was added. The reaction mixture was heated under reflux for $2 \mathrm{~h}$. after cooling precipitate was filtered, dried and recrystallized from the appropriate solvent to give $\mathbf{3 a}, \mathbf{b}, \mathbf{5 , 6 , 8}$ and $\mathbf{9}$, respectively

\section{2-Acetamido-N-(4-nitrophenyl)-3-(naphthalen-2-yl)but-2-enamide (3a).}

$\operatorname{IR}\left(\mathrm{cm}^{-1}, \mathrm{v}\right):$ 1309, $1552\left(\mathrm{NO}_{2}\right) 1663(\mathrm{br}, 2 \mathrm{C}=\mathrm{O}), 3354,3472(2 \mathrm{NH})$ groups; ${ }^{1} \mathrm{H}-\mathrm{NMR}$ $\left(\mathrm{CHCl}_{3}, \delta, \mathrm{ppm}\right): 2.38,2.67\left(2 \mathrm{~s}, 6 \mathrm{H}, 2 \mathrm{CH}_{3}\right), 6.59,6.64(2 \mathrm{~s}, 2 \mathrm{H}, 2 \mathrm{NH}$, exchangeable with $\left.\mathrm{D}_{2} \mathrm{O}\right), 7.25-8.07(\mathrm{~m}, 10 \mathrm{H}, \mathrm{ArH}), 8.3\left(\mathrm{~s}, 1 \mathrm{H}, \mathrm{C}_{-1} \mathrm{ArH}\right)$.

\section{2-Acetamido-N-(4-chlorphenyl)-3-(naphthalen-2-yl)but-2-enamid (3b).}

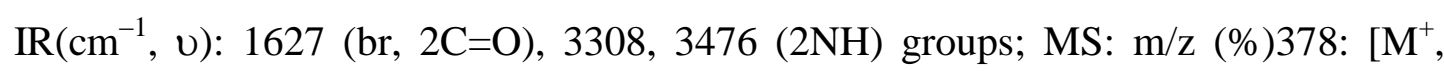

\section{2-Acetamido-N,N-diethyl-3-(naphthalen-2-yl)but-2-enamide (5).}

$\operatorname{IR}\left(\mathrm{cm}^{-1}, \quad \mathrm{v}\right): 1667 \quad(\mathrm{br}, 2 \mathrm{C}=\mathrm{O}), \quad 3399, \quad(\mathrm{NH}+$ enaolic $\mathrm{OH})$ groups; ${ }^{1} \mathrm{H}-\mathrm{NMR}$ (DMSO-d $\left.\mathrm{d}_{6}, \delta, \mathrm{ppm}\right): 1.01\left(\mathrm{t}, \mathrm{J}=7.5 \mathrm{~Hz}, 6 \mathrm{H}, 2 \mathrm{CH}_{3}\right), 1.67,2.72\left(2 \mathrm{~s}, 6 \mathrm{H}, 2 \mathrm{CH}_{3}\right), 3.51$ $\left(\mathrm{q}, \mathrm{J}=7.5 \mathrm{~Hz}, 4 \mathrm{H}, 2 \mathrm{CH}_{2}\right), 7.60-8.01(\mathrm{~m}, 6 \mathrm{H}, \mathrm{ArH}), 8.31\left(\mathrm{~s}, 1 \mathrm{H}, \mathrm{C}_{-1} \mathrm{ArH}\right), 8.96(\mathrm{~s}, 1 \mathrm{H}, \mathrm{NH}$, exchangeable with $\mathrm{D}_{2} \mathrm{O}$ ).

N-1-Morpholino-3-(naphthalen-2-yl)-1-oxobut-2-en-2-yl)acetamide (6).

$\mathrm{IR}\left(\mathrm{cm}^{-1}, \mathrm{v}\right): 1669(\mathrm{br}, 2 \mathrm{C}=\mathrm{O}), 3389(\mathrm{NH}+$ enolic $\mathrm{OH})$ groups; ${ }^{1} \mathrm{HNMR}\left(\mathrm{CHCl}_{3}, \delta\right.$ ppm): 2.22, $2.54\left(2 \mathrm{~s}, 6 \mathrm{H}, 2 \mathrm{CH}_{3}\right), 3.24-3.70\left(\mathrm{~m}, 8 \mathrm{H}, 4 \mathrm{CH}_{2}\right.$, morpholino), 7.55-8.02 (m, $6 \mathrm{H}$, ArH), 8.30 (s, 1H, $\left.\mathrm{C}_{-1} \mathrm{ArH}\right), 9.43\left(\mathrm{br}, 1 \mathrm{H}, \mathrm{NH}\right.$, exchangeable with $\left.\mathrm{D}_{2} \mathrm{O}\right)$.

1,2-Dihydro-3-methyl-5-(1-(naphthalen-2-yl)ethylidene)-1,2,4-triazin-6(5H)-one (8).

$\operatorname{IR}\left(\mathrm{cm}^{-1}, \mathrm{v}\right): 1676(\mathrm{C}=\mathrm{O}), 3123(2 \mathrm{NH})$ groups; $\mathrm{MS}: \mathrm{m} / \mathrm{z}(\%): 265\left[\mathrm{M}^{+}, 18.67\right)$. 


\section{2-Acetamido-N-(4-aminophenyl)-3-naphthalen-2-yl)but-2-enamide (9).}

$\mathrm{IR}\left(\mathrm{cm}^{-1}, \mathrm{v}\right): \quad$ 1664(br,2C=O), 3167, 3301, 3445( $\left.\mathrm{NH}_{2} / \mathrm{NH}\right) \quad$ groups. ${ }^{1} \mathrm{H}-\mathrm{NMR}\left(\mathrm{CHCl}_{3}, \delta, \mathrm{ppm}\right): 2.22,2.46\left(2 \mathrm{~s}, 6 \mathrm{H}, 2 \mathrm{CH}_{3}\right), 3.48\left(\mathrm{~s}, 2 \mathrm{H}, \mathrm{NH}_{2}, \mathrm{D}_{2} \mathrm{O}\right.$ exchangeable), 7.55-8.04 (m, 10H, ArH), 8.47 (s, 1H, $\left.\mathrm{C}_{-1} \mathrm{ArH}\right), 9.44\left(\mathrm{~s}, 2 \mathrm{H}, 2 \mathrm{NH}\right.$, exchangeable with $\left.\mathrm{D}_{2} \mathrm{O}\right)$.

General procedure for the preparation of the compounds $4 a, b$ and 10.

\section{Procedure 1:}

A solution of compound $2(0.01 \mathrm{~mol})$ in acetic acid $(20 \mathrm{~mL})$ was treated with $1 \mathrm{gm}$ of fused sodium acetate and $(0.01 \mathrm{~mol})$ of (p-nitroaniliine, $p$-chloroaniline, or $p$-aminoaniline). The mixture was heated for $3 \mathrm{~h}$, the solid obtained was collected and recrystallized to give $\mathbf{4 a , b}$ and 10.

\section{Procedure 2:}

A mixture of (compound 3a,3b, or 9) $(0.01 \mathrm{~mol})$ and $1 \mathrm{gm}$ of fused sodium acetate in acetic acid $(20 \mathrm{~mL})$ was refluxed for $2 \mathrm{~h}$. After cooling, the product formed was filtered off, air dried and recrystallized to give $\mathbf{4 a}, \mathbf{b}$ and $\mathbf{1 0 .}$

\section{1-(4-Nitrophenyl)-2-methyl-4-(1-naphthalen-2-yl)ethylidene)- $1 H$-imidazole-5(4H)-one} (4a).

IR $\left(\mathrm{cm}^{-1}, \mathrm{v}\right): 1677(\mathrm{C}=\mathrm{O}), 1619(\mathrm{C}=\mathrm{N}), 1505,1332\left(\mathrm{NO}_{2}\right) ;{ }^{1} \mathrm{H}-\mathrm{NMR}\left(\mathrm{DMSO}_{\mathrm{d}}, \delta\right.$, ppm): 1.86, $\left.2.71\left(2 \mathrm{~s}, 6 \mathrm{H}, 2 \mathrm{CH}_{3}\right), 7.51-8.01\right)(\mathrm{m}, 10 \mathrm{H}, \mathrm{ArH}), 8.11\left(\mathrm{~s}, 1 \mathrm{H}, \mathrm{C}_{-1}\right.$ naphthalene).

1-(4-Chlorophenyl)-2-methyl-4-(1-(naphthalen-2-yl) ethylidene)-1H-imidazol-5(4H)-one (4b).

IR $\left(\mathrm{cm}^{-1}, \mathrm{v}\right): 1629(\mathrm{C}=\mathrm{N}) ; 1672(\mathrm{C}=\mathrm{O}), \mathrm{MS}: \mathrm{m} / \mathrm{z}(\%): 360\left[\mathrm{M}^{+}, 5.21\right]$.

1-(4-Aminophenyl)-2-methyl-4-(1-(naphthalene-2-yl)ethylidene)-1H-imidazol-5(4H)one (10).

$\operatorname{IR}\left(\mathrm{cm}^{-1}, \quad\right.$ v) $1632(\mathrm{C}=\mathrm{N}), \quad 1669 \quad(\mathrm{C}=\mathrm{O}), \quad 3180, \quad 3305 \quad\left(\mathrm{NH}_{2}\right)$ groups; ${ }^{1} \mathrm{H}-\mathrm{NMR}\left(\mathrm{DMSO}-\mathrm{d}_{6}, \delta \mathrm{ppm}\right): 1.66,2.24\left(2 \mathrm{~s}, 6 \mathrm{H}, 2 \mathrm{CH}_{3}\right), 7.25\left(\mathrm{~s}, 2 \mathrm{H}, \mathrm{NH}_{2}\right.$, exchangeable with $\left.\mathrm{D}_{2} \mathrm{O}\right), 7.57-8.02(\mathrm{~m}, 10 \mathrm{H}, \mathrm{ArH}), 8.46\left(\mathrm{~s}, 1 \mathrm{H}, \mathrm{C}_{-1}\right.$ naphthalene).

\section{2-Acetamido-3-(naphthalen-2-yl)but-2-enehydrazide (7).}

A mixture of oxazolinone $2(0.01 \mathrm{~mol})$ and hydrazine hydrate $(0.015 \mathrm{~mol})$ in ethanol $(20 \mathrm{~mL})$ was stirred at room temperature for $2 \mathrm{~h}$. The product obtained was filtered and recrystallized to give 7. IR $\left(\mathrm{cm}^{-1}, \mathrm{v}\right): 1691(\mathrm{br}, \mathrm{C}=\mathrm{O}), 3131,3210,3363\left(\mathrm{NH}_{2} / \mathrm{NH}\right)$ groups; ${ }^{1} \mathrm{H}-\mathrm{NMR}\left(\mathrm{CHCl}_{3}, \delta \mathrm{ppm}\right): 1.63,2.73\left(2 \mathrm{~s}, 6 \mathrm{H}, 2 \mathrm{CH}_{3}\right), 3.72\left(\mathrm{~s}, 2 \mathrm{H}, \mathrm{NH}_{2}\right.$, exchangeable with $\left.\mathrm{D}_{2} \mathrm{O}\right)$. 7.52-7.92 (m, 6H, ArH), $8.25\left(\mathrm{~s}, 1 \mathrm{H}, \mathrm{C}_{-1}\right.$ naphthalene).9.25, 9.51(2s, $2 \mathrm{H}, 2 \mathrm{NH}$, exchangeable with $\mathrm{D}_{2} \mathrm{O}$ ).

1-[(4-Tolylsulfonamido)phenyl)]-2-methyl-4-(1-(naphthalen-2-yl)ethylidene)-1Himidazol-5(4H)-one (11).

A mixture of compound $\mathbf{1 0}(0.01 \mathrm{~mol})$ and p-toluene sulphonyl chloride $(0.01 \mathrm{~mol})$ in benzene/pyridine $(20 / 10 \mathrm{~mL})$ was heated under reflux for $3 \mathrm{~h}$. The reaction mixture was cooled and the product obtained was filtered off, dried and recrystallized to give 11.

IR $\left(\mathrm{cm}^{-1}, \mathrm{v}\right): 1187,1361\left(\mathrm{SO}_{2}-\mathrm{N}\right), 1620(\mathrm{C}=\mathrm{N}) 1671(\mathrm{C}=\mathrm{O}), 3432(\mathrm{NH})$ groups; ${ }^{1} \mathrm{H}-$ $\mathrm{NMR}\left(\mathrm{CHCl}_{3}, \delta, \mathrm{ppm}\right), 1.22,1.52,2.57\left(3 \mathrm{~s}, 9 \mathrm{H}, 3 \mathrm{CH}_{3}\right), 7.25(\mathrm{~s}, 1 \mathrm{H}, \mathrm{NH}$, exchangeable with $\left.\mathrm{D}_{2} \mathrm{O}\right), 7.55-7.97(\mathrm{~m}, 14 \mathrm{H}, 3 \mathrm{ArH}), 8.47\left(\mathrm{~s}, 1 \mathrm{H}, \mathrm{C}_{-1}\right.$ naphthalene). 
1-[4-(4-Chlorobenzylidine)aminophenyl)]-2-methyl-4-(1-(naphthalen-2-yl) ethylidine)1H-imidazole-5(4H)-one 12.

To a solution of compound $\mathbf{1 0}(0.0 \mathrm{~mol})$ in ethanol, 4-chloro- benzaldehdye $(0.01$ mol) was added followed by addition of 2-3 drops glacial acetic acid, then, the reaction mixture was heated under reflux temperature for $2 \mathrm{~h}$, the solid obtained after cooling was filtered, dried and recrystallized to give 12. IR $\left(\mathrm{cm}^{-1}, \mathrm{v}\right): 1599(\mathrm{HC}=\mathrm{N}-), 1664(\mathrm{C}=\mathrm{O})$ groups; ${ }^{1} \mathrm{H}-\mathrm{NMR}\left(\mathrm{DMSO}-\mathrm{d}_{6}, \delta\right.$, ppm): 2.00, $2.71\left(2 \mathrm{~S}, 6 \mathrm{H}, 2 \mathrm{CH}_{3}\right), 7.46-7.98$ (m, 14H, 3ArH), 8.67(s, $1 \mathrm{H}, \mathrm{C}_{-1}$ naphthalene), $9.83(\mathrm{~s}, 1 \mathrm{H}, \mathrm{CH}=\mathrm{N}-$ ).

\section{1-(Naphthalen-2-yl)ethylidenemalononitrile 13.}

A mixture of compound $\mathbf{1 0}(0.01 \mathrm{~mol})$ and malononitrile was heated under refluxed in ethanolic piperidine for $2 \mathrm{~h}$. the solid obtained after cooling was filtered, dried and recrystallized to give 12. IR $\left(\mathrm{cm}^{-1}, \mathrm{v}\right): 1589(\mathrm{C}=\mathrm{C}), 2210(2 \mathrm{C} \equiv \mathrm{N}) ; \mathrm{MS}: \mathrm{m} / \mathrm{z}(\%): 218\left(\mathrm{M}^{+}\right.$, 100).

\section{2-Amino-4-(naphthalen-2-yl)thiophen-3-carbonirtrile (15a).}

\section{Procedure A:}

A solution of ethylidenemalononitrite $13(0.01 \mathrm{~mol})$ and sulfur powder $(0.01 \mathrm{~mol})$ in ethanol $(30 \mathrm{~mL})$ containing few drops of piperidine, was refluxed for $3 \mathrm{~h}$. The solid product was filtered and recrystallized to give 15a.

\section{Procedure B: For preparation of 15a and 15b:}

A mixture of compound $1(0.01 \mathrm{moL})$, malononitrile or ethyl cyanoacetate $(0.01$ $\mathrm{moL})$ and sulfur powder $(0.01 \mathrm{moL})$ was refluxed in ethanol containing few drops of triethylamine for $2 \mathrm{~h}$. the solid formed after filtration and cooling was crystallized to give 15a and $\mathbf{1 5 b}$ respectively.

\section{2-Amino-4-(naphthalen-2-yl)thiophene-3-carbonitrile (15a).}

IR $\left(\mathrm{cm}^{-1}, \mathrm{v}\right): 2206(\mathrm{C} \equiv \mathrm{N}), 3202,3322\left(\mathrm{NH}_{2}\right)$ groups; ${ }^{1} \mathrm{H}-\mathrm{NMR}\left(\mathrm{DMSO}-\mathrm{d}_{6}, \delta, \mathrm{ppm}\right)$ : 3.74(br., $2 \mathrm{H}, \mathrm{NH}_{2}$, exchangeable with $\mathrm{D}_{2} \mathrm{O}$ ), 6.68 (s, $1 \mathrm{H}, \mathrm{C}_{-5}$ thiophene), 7.15-7.99 (m, 6H, ArH), 8.68 (s, 1H, $\mathrm{C}_{-1}$ naphthalene).

Ethyl-2-amino-4-(naphthalen-2-yl) thiophen-3-carboxylate (15b).

IR $\left(\mathrm{cm}^{-1}, \mathrm{v}\right): 1721(\mathrm{C}=\mathrm{O}), 3200,3321\left(\mathrm{NH}_{2}\right)$ groups; ${ }^{1} \mathrm{HNMR}\left(\mathrm{DMSO}-\mathrm{d}_{6}, \delta, \mathrm{ppm}\right): 1.49(\mathrm{t},=$ $\left.8.5 \mathrm{~Hz}, 3 \mathrm{H}, \mathrm{CH}_{3}\right) 3.39$ (br, $2 \mathrm{H}, \mathrm{NH}_{2}$, exchangeable with $\left.\mathrm{D}_{2} \mathrm{O}\right), 3.75\left(\mathrm{q}, \mathrm{J}=7.5 \mathrm{~Hz}, 2 \mathrm{H}, \mathrm{CH}_{2}\right)$, 6.67(s, 1H, $\mathrm{C}_{-5}$ thiophene), 7.60-8.01 (m, 6H, ArH), 8.68 (s, 1H, $\mathrm{C}_{-1}$ naphthalene).

\section{5-(Naphthalen-2yl)thieno[2,3-d]pyrimidin-4(3H)-one (16).}

Compound 15a $(0.01 \mathrm{~mol})$ was heated under reflux temperature in $20 \mathrm{~mL}$ formic acid for $6 \mathrm{~h}$. The reaction mixture was cooled, poured into water, filtered dried and the residue was recrystallized to give $\mathbf{1 6}$.

IR $\left(\mathrm{cm}^{-1}, \mathrm{v}\right): 1596(\mathrm{C}=\mathrm{N}), 1697(\mathrm{C}=\mathrm{O}), 3211(\mathrm{NH}) ;{ }^{1} \mathrm{H}-\mathrm{NMR}\left(\mathrm{DMSO}-\mathrm{d}_{6}, \delta, \mathrm{ppm}\right): 6.7(\mathrm{~s}$, $\left.1 \mathrm{H}, \mathrm{C}_{-6} \mathrm{H}\right), 7.20-7.97(\mathrm{~m}, 7 \mathrm{H}, \mathrm{ArH}+\mathrm{NH}), 8.15\left(\mathrm{~s}, 1 \mathrm{H}, \mathrm{C}_{-2} \mathrm{H}\right), 8.31$ (s, 1H, $\mathrm{C}_{-1}$ naphthalene).

Ethyl N-[4-(naphthalen-2yl)]-3-cyanothieno-2-yl) methanimidate (17).

A mixture of compound $\mathbf{1 5 a}(0.01 \mathrm{~mol})$ and $20 \mathrm{~mL}$ triethyl orthoformate was heated under reflux temperature for $4 \mathrm{~h}$, then evaporated and the residue was recryustallized to give compound 17. 
IR $\left(\mathrm{cm}^{-1}, \mathrm{v}\right): 2211(\mathrm{C} \equiv \mathrm{N}) ;{ }^{1} \mathrm{H}-\mathrm{NMR}\left(\mathrm{DMSO}_{\mathrm{d}}, \delta, \mathrm{ppm}\right): 1.23\left(\mathrm{t}, 8.5 \mathrm{~Hz}, 3 \mathrm{H}, \mathrm{CH}_{3}\right)$, $3.75\left(\mathrm{q}, \mathrm{J}=7.50,2 \mathrm{H}, \mathrm{CH}_{2}\right), 6.71(\mathrm{~s}, 1 \mathrm{H}$, thiophene), 7.25-7.81 (m, $6 \mathrm{H}, \mathrm{ArH}), 8.21\left(\mathrm{~s}, 1 \mathrm{H}, \mathrm{C}_{-}\right.$ naphthalene), $8.51(\mathrm{~s}, 1 \mathrm{H}, \mathrm{N}=\mathrm{CHOEt})$.

\section{4-Imino-5-(naphthalen-2yl)thieno[2,3-d]pyrimidin-3-ylamine (18).}

A mixture of compound 17 (0.01) dissolved in $20 \mathrm{~mL}$ of absolute ethanol and $3 \mathrm{~mL}$ of hydrazine hydrate $(99 \%)$, was stirred for $1 \mathrm{~h}$ at room temperature. The solid that formed was filtered, washed with a little amount of methanol dried, and recrystallized to give compound 18. IR $\left(\mathrm{cm}^{-1}, \mathrm{v}\right): 1645(\mathrm{C}=\mathrm{N}), 3111,3220,3250\left(\mathrm{NH}_{2} / \mathrm{NH}\right)$ groups.; $\mathrm{MS}: \mathrm{m} / \mathrm{z}$ $(\%): 292\left(\mathrm{M}^{+}, 100\right)$

\section{5-(Naphthalene-2-yl)thieno[2,3-d]pyrimidin-4-yl-hydrazine (19):}

\section{Method A:}

Compound $17(0.01 \mathrm{~mol})$ was dissolved in $20 \mathrm{~mL}$ absolute ethanol, then $2 \mathrm{~mL}$ of hydrazine hydrate $(99 \%)$ were added, and the reaction mixture was heated under reflux temperature for $3 \mathrm{~h}$; it was evaporated and the residue was recrystallized to give 19 . IR $\left(\mathrm{cm}^{-1}\right.$, u): 3180, 3210, $3310\left(\mathrm{NH}_{2} / \mathrm{NH}\right)$; $\mathrm{MS}: \mathrm{m} / \mathrm{z}(\%): 292\left(\mathrm{M}^{+}, 71\right)$

\section{Method B: Isomerization of 18 to 19.}

Compound 18 (0.01 mol) was dissolved in $20 \mathrm{~mL}$ ethanol and then drops of hydrazine hydrate were added, then the reaction mixture was heated under reflux temperature for $2 \mathrm{~h}$ and evaporated under reduced pressure to give compound 19. Product 19 which was obtained from this isomarization is identical in all respects (physical and spectral data) to those prepared by method A.

\section{Antioxidant Assay:}

The antioxidant activity of extract was determined by the DPPH free radical scavenging assay method (Sonia, et al., 2008) in triplicate and average values were considered.

\section{DPPH antioxidant assay}

Freshly prepared $(0.004 \% \mathrm{w} / \mathrm{v})$ methanol solution of 2,2-diphenyl-1-picrylhydrazyl (DPPH) radical was prepared and stored at $10^{\circ} \mathrm{C}$ in the dark. A methanol solution of the test compound was prepared. A 40ul aliquot of the methanol solution was added to $3 \mathrm{ml}$ of DPPH solution. Absorbance measurements were recorded immediately with a Milton Roy Spectronic $201 \mathrm{UV}$-visible spectrophotometer. The decrease in absorbance at $515 \mathrm{~nm}$ was determined continuously, with data being recorded at $1 \mathrm{~min}$ intervals until the absorbance stabilized (16 min). Tocopherol was used as a reference standard and dissolved in distilled water to make the stock solution with the same concentration. The absorbance of the DPPH radical without antioxidant was also measured as control and 95\% methanol was used as blank. All the determinations were performed in three replicates and averaged.

$\%$ Scavenging of the DPPH free radical was measured using the following equation: $\%$ DPPH radical-scavenging $=[($ Absorbance of control - Absorbance of test Sample $) /$ (Absorbance of control)] x 100 . 


\section{REFERENCES}

Abdelrazek, F.M.; Ead, H.A. (1996): Phosphorus, Sulfur and Silicaon, 119, 271-277.

Achar, K.C.S.; Hosamani, K.M.; Seetharamareddy, H.R.; (2010): 45, 2048-2054.

Chuu, J.-J.; Liu, J. M.; Tsou, M. H.; Huang, C.-L.; Chen, C.-P.; Wang, H.-S.; Chen, C.T. (2007): Effects of paclitaxel and doxorubicin in histocultures of hepatocelular carcinomas. J. Biomed. Sci., 14, 233-244.

De Rycker, M.; Rigoreau, L.; Dowding, Parker, P.J. (2009): A high content, cell-based screen identifies micropolyin, a new inhibitor of microtubule dynamics; chem.. Biol. Drug Des. 73, 599-610.

Gewald, K.G. E. (1965): Chem. Ber., 98, 357.

Hafez, H.N.; Hussein, H.A.R.; El-Gazzar, A.B.A.(2010): Synthesis of substituted thieno(2,3d)pyrimidine-2,4-dithiones and their S-glycoside analogues as potential antivral and antibacterial agents; European Journal of medicinal chemistry, 45, 4026-4034.

Hait, W. n. Hambley, T.W. (2009): Targeted Cancer therapeutics; Cancer Res., 69, 12631267.

Hegab Mohamed I., Hassan Nasser A., Rashad Aymn E. , Fahmy Afaf A. and AbdelMegeid Farouk M. E. (2007): Synthesis, Reactions, and Antimicrobial Activity of Some Fused thieno[2,3-d]pyrimidine Derivatives. Phosphorus, Sulfur, and Silicon and the Related Elements, 182, 1535-1556.

Hessien, S.A.; Kadah, M.S.; Marzouk, N.A. (2009): Synthesis of some Novel thiazole, thiophene containing topsyl moiety; Al-Azhar Bull. Sci., 20(1), 139-150.

Ingle, R.G.; Magar, D.D.; (2011): Heterocyclic chemistry of benzimidazoles and potential activities of derivatives; Int. J. Drug Res. Tech. 1(1), 26-32.

Kanthou, C.; Tozer, G.M. (2009): Microtubule depolymerizing vascular disrupting agents: novel therapeutic agents for oncology and other pathologies; Int. J. Exp. Pathol, 90, 284-294.

Kumari, S.; Pramod, K.S.; Nitin, K.; (2010): Imidazole and its biological activities; Der. Chemica Sinica, 1(3): 36-47.

Li, W. T.; Hwang, D. R.; Chen, C. P.; Shen, C. W.; Huang, C. L.; Chen, T. W.; Lin, C. H.; Chang, Y. L.; Chang, Y. Y.; Lo, Y. K.; Tseng, H. Y.; Lin, C. C.; Song, J. S.; Chen, H. C.; Chen, S. J.; Wu, S. H.; Chen, C.T. (2003): Synthesis and biological evaluation of $\mathrm{N}$-heterocyclic indolyl glycoxylamides as pro. active anticancer agents. J. Med. Chem., 46, 1706-1715.

Liberatore, A. M.; Coulomb, H.; Pons, D.; Dutruel, O.; Kasprzyk, P. G.; Carlson, M.; Nelson, A. S.; Newman, S. P.; Stengel, C.; Auvray, P.; Hesry, V.; Foll, B.; Narboux, N.; Morlais, D.; Le Moing, M.; Bernetiere, S.; Dellile, R.; Camara, J.; Ferrandis, E.; Bigg, D. C.; Pr_evost, G. P. (2008): IRC-083927 is a new tubulin binder that inhibits growth of human tumor cells resistant to standard tubulin-binding agents. Mol. Cancer Ther., 7, 2426-2434.

Maddila, S.; Palakondu, L.; Chunduri, V. (2010): Synthesis antibacterial, antifungal and antioxidant activity studies on 2-benzylthio-and-2-benzylsulfonyl- $1 \mathrm{H}$ imidazoles; Der Pharma Lettre, 2(4): 393-402. 
Mahboobi, S.; Sellmer, A.; Hcocher, H.; Eichhorn, E.; Bcar, T.; Schmidt, M.; Maier, T.; Stadlwieser, J. F.; Beckers, T. L. (2006): [4-(Imidazol-1-yl)thiazol-2yl]phenylamines. A novel class of highly potent colchicine site binding tubulin inhibitors: synthesis and cytotoxic activity on selected human cancer cell lines. J. Med. Chem., 49, 5769-5776.

Marzouk Nermin A., Shamroukh Ahmed H., Al-Saadny Abeer H., Micky Jehan A. and Abd EI-Megeid Farouk M. E. (2011): Synthesis, reactions and anticancer evaluation of some indenothieno pyrimidine derivatives Az. J. Pharm. Sci. 43, 148-160.

Mohamed, M.A.; Rashad, A.E.; Zaki, M.E.A.; Fatahala, S.S. (2005): Act Pharm., 55, 237249.

Nanda, S.P.; Padhy, A.K.; Dey, R.K.; (2010): Synthesis of some benzothiazolyl and imidozole derivatives and evaluation of antibacterial activities, J. Chem. Pharn. Res. 2(4): 785-792.

Rajasekaran, S.; GopalKrishna, R. Sanjay, P.; Gurpreet, K.V.; Gurpreet, S.S.; (2010): Synthesis and in vitro study of biological activity of 2,3-substituted quinazolin4(3H)-ones; J. Chem. Pharm. Res. 2(2): 462-468.

Rashad, A.E.; Heikel, O.A.; El-Nezhawy, A.O.H.; Abdel-Megeid, F.M.E. (2005): Heteroatom Chem., 16, 226-234.

Sanchila, B.; Zaihra, A.; Pramod, R.D.; Nitin, K. (2010): Recent advancement in imidazole as anticancer agents; Der Pharmacia Sinica, 1(3), 172-182.

Shailesh, P.Z.; Badmanaban, R.; Dhrubo, J.S.; Chhaganbhai, N.P.; (2012): Synthesis and biological evaluation of 2,4,5-triphenyl-1-H-imidazole-1-yl derivatives; Journal of applied pharmaceutical science, 02(07): 202-208.

Shamrokh, A.H., Marzouk, N.A., Al-Saadny, A.H., Micky, J.A., and Abdel-Megeid, F.M.E.; (2010): Anti-H5N1 Evaluation of some newly synthesized indenthienopyrimidine derivatives, World J. Chem. 5(1): 73-43.

Sonia, G.; Kumar, M.P.; Raja, A.C.; Ravi, T.K.; (2008): Acta Pharm., 58, 119.

Soroor, S.; Seyed, S.S. (2012): Affectivity of the imidazole anticancer drug on the diagnosis of the cancers by using quantum chemical methods, $4^{\text {th }}$ international conference on bioinformatics and biomedical technology IPCBEE 29, 187-190.

Wen-Tai, L.; Der-Ren, H.; Jen-Shin, S.; Ching-Ping, Ch. (2010): Synthesis and biological activities of 2-amino-1-arylidenamino imidazoles as orally active anticancer; J. Med. Chem. 53, 240-2417. 
تشييد، تفاعلات ومضادات الأكسدة لبعض الحلقات الغير متجانسة الحلقة الجديدة المشيدة

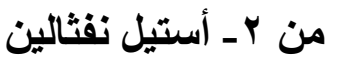

نادية محمود طه ، إيناس إسلام ، نرمين عبدالله مززوق، انتصار محمد و عبير علي

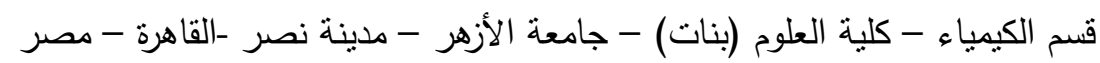

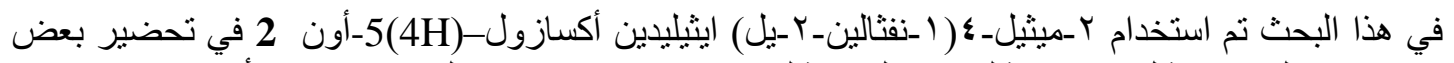

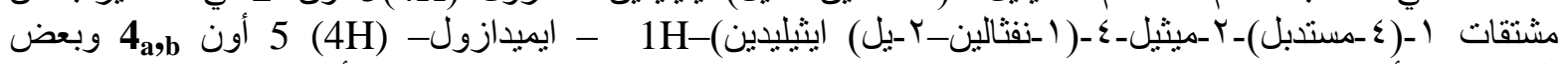
المشتقات الأخرى 3-12

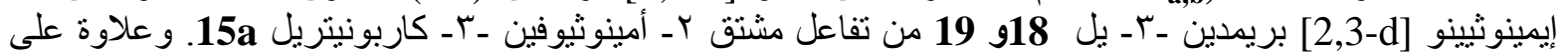
هذا تم اختبار النشاط المضاد للأكسدة و أعطت بعض هذه المركبات فاعلية كمضادات للاكسدة. 